\title{
Torsional Buckling of Thin-Walled Columns with Transverse Stiffeners: Analytical Studies
}

\author{
Trung Hoang ${ }^{1 *}$, Sándor Ádány ${ }^{1}$ \\ 1 Department of Structural Mechanics, Faculty of Civil Engineering, Budapest University of Technology and Economics, $\mathrm{H}-1111$ \\ Budapest, Müegyetem rkp. 3., Hungary \\ * Corresponding author, e-mail: hoang.trung@epito.bme.hu
}

Received: 17 October 2019, Accepted: 16 January 2020, Published online: 09 March 2020

\begin{abstract}
In this paper the pure torsional buckling of thin-walled column members is investigated, with a special focus on the effect of transverse plate elements, such as end-plates or transverse stiffeners. The linear buckling problem is aimed to solve analytically, therefore the necessary (differential) equations are first established. For some simple problems, namely doubly-symmetric l-sections with pinnedpinned or clamped-clamped supports and with rectangular stiffeners or end-plates, closed formulae are derived to calculate the critical force. It is shown that the transverse elements have two effects: the direct effect is due to the deformation of the transverse elements, while the indirect effect is that the transverse elements modify the longitudinal distribution of the member's displacements. It is also shown how the stiffener-to-member connection influences the results. The analytical solutions are discussed by several numerical examples: the results from the derived formulae are compared to results from shell finite element buckling analyses.
\end{abstract}

\section{Keywords}

torsional buckling, thin-walled member, transverse stiffeners, end-plates

\section{Introduction}

Thin-walled members appear in many structural engineering applications, most frequently made of steel. If subjected to torsion, the behavior of thin-walled members is complicated, characterized by both Saint-Venant torsion (which induces shear stresses only) and warping (which induces axial and shear stresses). The classic description of the problem can be found in textbooks $[1,2]$. Though the underlying differential equation (DE) is known, its practical handling is challenging. Exact solution of the $\mathrm{DE}$ is difficult even for the simplest members. Numerical (approximate) solution is possible, e.g. by using the finite element method. If beam finite elements are used, minimum 7 degrees of freedom (DOF) per node are necessary due to the torsion: the classic 6 displacement DOF must be supplemented by a warping DOF. Such special beam element is not always included in commercial FEM software implementations, and even if included, the warping DOF induces practical questions (e.g., at supports, at joints) that are not easy to correctly respond.

In thin-walled members buckling is always important. When a structural member buckles, various buckling types are distinguished, depending on the loading of the member and depending on the displacements involved in the buckling. Even if we limit our investigations to buckling types characterized by practically rigid cross-sections, various buckling types can be defined. In the case of columns flexural, pure torsional and flexural-torsional buckling types are usually distinguished. In the case of beams, the buckling is termed lateral-torsional buckling. With the exception of flexural buckling of columns (with symmetric cross-sections), in all the other buckling types torsion is involved in the buckled shapes. Though from practical aspect the pure torsional buckling is rarely governing, this is the simplest form of buckling with torsion, therefore the proper understanding of pure torsional buckling can help in solving flexural-torsional or lateral-torsional buckling, too. Research on pure torsional buckling of columns is hardly reported recently, though some specific problems are addressed [3-6]. In this paper pure torsional buckling of columns is investigated, with a special focus on the effect of transverse plate elements.

In thin-walled members in many cases transverse plate elements are applied. Such transverse plate element may appear as an end-plate, a gusset plate, or transverse 
stiffener. End-plates and gusset plates are applied in order to facilitate the connection between various structural members (i.e., a beam to a column), while stiffeners are typically applied in order to increase the resistance to buckling of slender plates. Transverse stiffeners are known to be effective against shear buckling of a web or web crippling at supports. Though stiffeners, end-plates and gusset plates have different roles, they might have different shapes, etc., they have very similar mechanical effect on the torsional behavior of members. Thus, the term "transverse stiffener" will mostly be used in this paper, but in a general meaning.

While it is reasonable to assume that the presence of transverse stiffeners has negligible effect on the flexural behavior of the member, the transverse stiffeners definitely affect the torsional behavior. The effect of end-plates on the warping fixity is well-known, reported and discussed e.g. in $[7,8]$, though without giving a proper theoretical explanation on how the geometrical and material parameters are related to the warping springs. In [9] the effect of transverse stiffeners is correctly described by some basic equations, but the equations then are not applied to solve practical problems or to discuss some tendencies.

In classic buckling solutions for pure torsional (or flexural-torsional, lateral-torsional) buckling the effect of transverse stiffeners is not considered. The strength enhancing effect of end-plates on flexural-torsional buckling of columns is studied e.g. in [10], by experiments and nonlinear finite element simulations. The effect of end-plates on lateral-torsional buckling of beams appears in several studies, including [11-13], but without any attempt to handle the problem analytically. An analytical solution for the lateral-torsional buckling of beams with transverse stiffeners is briefly reported in [14], but the reported approach seems to be more appropriate for beams with batten plates rather than for beams with transverse web stiffeners. According to the authors best knowledge analytical solution for torsional buckling of thin-walled columns/beams with directly considering transverse stiffeners or endplates is not yet reported. Analytical considerations hardly appear in the literature, which is especially true for pure torsional buckling. The goal of the research reported here, therefore, is to derive analytical solutions for the critical load to pure torsional buckling of thin-walled columns with transverse elements.

In the paper first the problem is described in a general way (Section 2). Since our aim is to have analytical solution, the problem is simplified, and doubly-symmetric I-section will only be discussed in a detailed way. The solution requires the displacement of the stiffeners, hence in Section 3 the stiffeners are studied. In Section 4 analytical solution is derived for the critical load to pure torsional buckling of clamped-clamped columns with transverse stiffeners. The analytical solution is discussed and the results are compared to shell finite element solutions. In Section 5 pinned-pinned columns are studied with end-plates and transverse stiffeners: first analytical formulae are derived, then numerical results are discussed in comparison with shell FE results. Finally, the main conclusions are summarized.

Though in this paper pure torsional buckling is investigated only, it is believed that the methodology and the results presented in this paper can be extended to other types of buckling with torsion, like lateral-torsional buckling of beams.

\section{Formulation of the problem, solution strategy 2.1 Description of the problem in general}

Let us consider a straight and prismatic thin-walled member with a length $L$. It is modelled as a one-dimensional element with cross-sections perpendicular to the member axis, i.e., beam-model is adopted. The cross-sections are assumed to be rigid, hence the displacements of the member are given by the displacement function of the system line. Classic beam theory is assumed, that is for the torsional behavior Vlasov's theory is applied (which can be regarded as the extension of classic Euler-Bernoulli beam theory). The material is isotropic and linearly elastic. The member is a column, loaded by two opposite axial forces at the member ends, uniformly distributed over the cross-section.

Transverse plate elements are assumed. We consider $n_{s t}$ stiffeners, the position of each is given by $x_{s t, i}\left(i=1, \ldots, n_{s t}\right.$; $\left.0 \leq x_{s t, i} \leq L\right) ; L$ is the length of the beam. The transverse plate elements are perpendicular to the member axis, otherwise they are arbitrary. The transverse plate elements are assumed to be thin so that the Kirchhoff-Love plate theory could be applied. The domain determined by the area of the $i$-th stiffener is denoted as $\Omega_{S, i}$. The transverse stiffeners are connected to the main member, in a general case through domain $\Omega_{L, i}$. The free edges of a stiffener plate are collectively denoted as domain $\Omega_{F, i}$. The member with the stiffeners and the characteristic domains are illustrated in Fig. 1.

The energy method is employed here, hence the displacement functions must be known or assumed. Since pure torsional buckling is investigated, the displacement of the member is described solely by the function of the twisting rotation $\theta(x)$. It has to satisfy the boundary conditions defined by the supports. In the case of energy method 

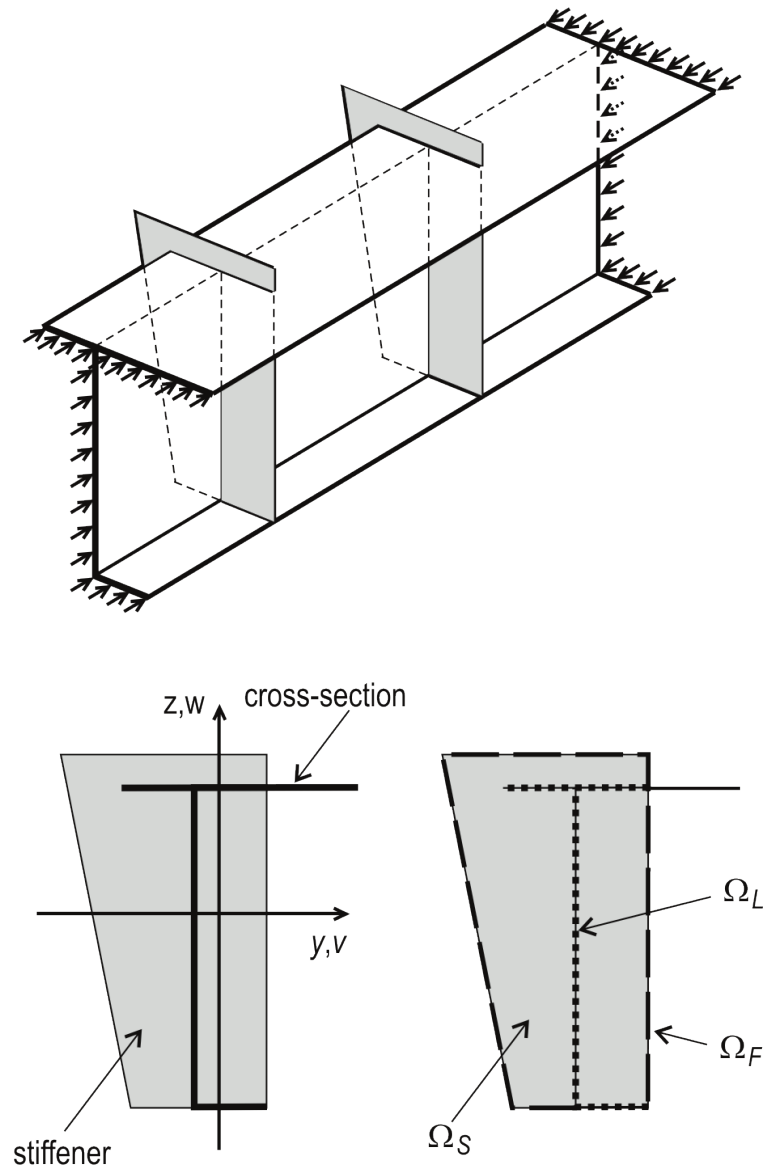

Fig. 1 A sample member and the characteristic domains of a stiffener

the function must be assumed; in many cases it is convenient to assume the function as a linear combination of simpler functions (e.g., trigonometric functions). Let us assume that the function is expressed as:

$\theta(x)=\sum_{1}^{k} c_{i} f_{i}(x)$

where $c_{i}(i=1, \ldots, k)$ are the unknown parameters and $f_{i}(x)$ are predefined functions.

By the twisting rotation function the $u, v$ and $w$ translational displacements (along the $x, y$ and $z$-axis, respectively) of any point of the member are determined as follows:

$v_{\theta}(x, y, z)=\theta(x) z$,

$w_{\theta}(x, y, z)=-\theta(x) y$,

$u_{\theta}(x, y, z)=\frac{\partial \theta(x)}{\partial x} y z$

The stiffeners are assumed to be thin plates, hence the $w_{s t, i}(y, z)$ displacement function of the $i$-th stiffener (where $\left.i=1, \ldots, n_{s t}\right)$ must satisfy the DE of the Kirchhoff-Love plate theory, plus it must satisfy the boundary conditions.
As well-known, the DE of the plate is as follows:

$$
\Delta \Delta w_{s t, i}(y, z)=\frac{\partial^{4} w_{s t, i}}{\partial y^{4}}+2 \frac{\partial^{4} w_{s t, i}}{\partial y^{2} \partial z^{2}}+\frac{\partial^{4} w_{s t, i}}{\partial z^{4}}=\frac{p_{s t, i}}{D_{s t, i}},
$$

where $D_{s t, i}$ is the plate stiffness, defined as:

$D_{s t, i}=\frac{E t_{s t, i}{ }^{3}}{12\left(1-v^{2}\right)}$,

and $t_{s t, i}$ is thickness of stiffener, $E$ is modulus of elasticity, $v$ is Poisson's ratio, while $p_{s t, i}$ is the load acting perpendicularly on the plate. In the actual column buckling problem this load is assumed to be zero, hence the right-hand-side of the DE is zero.

The $w_{s t, i}(y, z)$ function has to satisfy the boundary conditions. One part of the boundary conditions comes from the compatibility between the main member and the plate (over domain $\Omega_{L, i}$ ). The other part of the boundary conditions comes from the fact that the normal stress resultant (i.e., bending moment) along the plate free edges (i.e., over domain $\Omega_{F, i}$ ) is zero. Mathematically, therefore:

$$
\left\{\begin{array}{l}
w_{s t, i}(y, z)=u_{\theta}\left(x_{s t, i}, y, z\right), \text { if }(y, z) \in \Omega_{L, i} \\
\frac{\partial w_{s t, i}(y, z)}{\partial z}=\frac{\partial w_{\theta}}{\partial x \mid x=x_{s t, i}}, \text { if }(y, z) \in \Omega_{L, i} \\
\frac{\partial w_{s t, i}(y, z)}{\partial y}=\frac{\partial v_{\theta}}{\partial x \mid x=x_{s t, i}}, \text { if }(y, z) \in \Omega_{L, i} \\
\frac{\partial^{2} w_{s t, i}(y, z)}{\partial^{2} n}=0, \text { if }(y, z) \in \Omega_{F, i}
\end{array}\right.
$$

where $n$ denotes the direction perpendicular to the free edge. Eqs. (5)-(7) define a boundary value problem for each stiffener. Either we can find the strong solution by solving the differential equation, or we may find an approximate solution (e.g., by solving the weak formulation of the problem).

\subsection{Overview of the solution by the energy method}

To find the analytical solution for the critical load the energy method is followed: the total potential is expressed by some displacement parameters, then the theorem of stationarity of potential energy is used to find the equilibrium configuration. Since the primary aim here is to find critical load, in calculating the work of the external loading the displaced member is considered, by using quadratic approximation of the displacements. It is known that for simple straight columns the energy can be expressed with respect to the original undeformed configuration, i.e., the 
primary displacements-deformations can be disregarded: this is reflected in Eq. (4), and is also reflected in the calculation of the work of the external loading (as follows) where the second-order strain terms are considered only.

As far as the main member is concerned, the classic energy/work terms are applied. This means that the methodology followed here leads to the classic critical force formula of pure torsional buckling if no transverse stiffeners are added. However, the effect of the stiffeners are also considered. This effect is two-fold. Since the stiffener plates are connected to the main member the direct effect is that the stiffeners will displace/deform as soon as the main member is displaced/deformed; due to this deformation strain energy is accumulated in the stiffeners that energy is to be included in the potential energy function. However, there is a second effect, too: the stiffeners modify the longitudinal displacement function of the main member (as will be clearly demonstrated in Section 5).

The external potential is the negative of the work done by the loading on the (second-order) displacements. Since the only assumed loading is axial, we need the longitudinal second-order displacement only, which can be calculated from the second-order strains (i.e., relevant terms of the Green-Lagrange strain vector). The second-order strain, therefore:

$\varepsilon_{x}^{I I}=\frac{1}{2}\left(\left(\frac{\partial v_{\theta}}{\partial x}\right)^{2}+\left(\frac{\partial w_{\theta}}{\partial x}\right)^{2}\right)$.

By substituting Eqs. (1)-(3) into Eq. (8) we get:

$\varepsilon_{x}^{I I}=\frac{1}{2}\left(\frac{\partial \theta}{\partial x}\right)^{2}\left(z^{2}+y^{2}\right)$.

The longitudinal displacement (at a certain $y-z$ location in the cross-section):

$\Delta_{x}^{I I}=\int_{0}^{L} \varepsilon_{x}^{I I} d x$.

The external potential:

$\Pi_{\text {ext }}=-\int \frac{F}{A} \Delta_{x}^{I I} d A$,

where: $F$ is the applied axial force, and $A$ is the cross section area.

Substitute Eq. (10) then Eq. (9) into Eq. (11):

$$
\begin{aligned}
& \Pi_{e x t}=-\int \frac{F}{A} \frac{1}{2}\left(\frac{\partial \theta}{\partial x}\right)^{2} d x\left(z^{2}+y^{2}\right) d A, \\
& \Pi_{e x t}=-\frac{F}{2} \int_{0}^{L}\left(\frac{\partial \theta}{\partial x}\right)^{2} d x \int \frac{\left(z^{2}+y^{2}\right)}{A} d A .
\end{aligned}
$$

Note, if the shear center of the main member cross section coincides with the origin of the coordinate system then:

$$
\int \frac{\left(z^{2}+y^{2}\right)}{A} d A=r_{0}{ }^{2} .
$$

The internal potential is the accumulated strain energy. The strain energy in the main member is due to SaintVenant shear strains/stresses, and due to strains/stresses from warping. For the Saint-Venant strains/stresses:

$$
\begin{aligned}
& \kappa_{T}=\frac{\partial \theta}{\partial x} \rightarrow M_{T}=G I_{t} \kappa_{T}, \\
& \Pi_{\mathrm{int}}^{S-V}=\frac{1}{2} G I_{t} \int_{0}^{L} \kappa_{T}^{2} d x,
\end{aligned}
$$

where: $G$ is shear modulus, and $I_{t}$ is the Saint-Venant torsional constant.

For the warping strains/stresses:

$\kappa_{\omega}=\frac{\partial^{2} \theta}{\partial x^{2}} \rightarrow B=E I_{\omega} \kappa_{\omega}$

$\Pi_{\mathrm{int}}^{\text {warp }}=\frac{1}{2} E I_{\omega} \int_{0}^{L} \kappa_{\omega}^{2} d x$

where: $I_{\omega}$ is the second moment of the sectorial coordinate, calculated to the center of twisting rotation.

The strain energy in a stiffener plate can be calculated similarly, from the curvatures and stress resultants (i.e., moments). The curvatures are as follows:

$\kappa_{s t, i, y y}=-\frac{\partial^{2} w_{s t, i}}{\partial y^{2}}, \kappa_{s t, i, z z}=-\frac{\partial^{2} w_{s t, i}}{\partial z^{2}}, \kappa_{s t, i, y z}=-2 \frac{\partial^{2} w_{s t, i}}{\partial y \partial z}$.

The stress resultants are as follows:

$M_{s t, i, y y}=D_{s t, i}\left(\kappa_{s t, i, y y}+v_{i} \kappa_{s t, i, z z}\right)$,
$M_{s t, i, z z}=D_{s t, i}\left(\kappa_{s t, i, z z}+v_{i} \kappa_{s t, i, y y}\right)$,
$M_{s t, i, y z}=D_{s t, i}\left(1-v_{i}\right) \kappa_{s t, i, y z}$,

where: $D_{s t, i}$ is the plate stiffness, given by Eq. (5), and $v_{i}$ is the Poisson's ratio for the $i$-th stiffener.

The strain energy for a stiffener plate is:

$\Pi_{\mathrm{int}, i}^{s t}=\int_{\Omega_{s, i}} \frac{1}{2}\left(M_{s t, i, y y} \kappa_{s t, i, y y}+M_{s t, i, y z} \kappa_{s t, i, y z}+M_{s t, i, z z} \kappa_{s t, i, z z}\right) d y d z$.

After substituting Eqs. (19)-(20) into Eq. (21):

$$
\begin{aligned}
& \Pi_{\mathrm{int}, i}^{s t}=\frac{D_{s t, i}}{2} \int_{\Omega_{S, i}}\left[\left(\frac{\partial^{2} w_{s t, i}}{\partial y^{2}}+\frac{\partial^{2} w_{s t, i}}{\partial z^{2}}\right)^{2}\right. \\
& \left.-2\left(1-v_{i}\right)\left(\frac{\partial^{2} w_{s t, i}}{\partial y^{2}} \frac{\partial^{2} w_{s t, i}}{\partial z^{2}}-\left(\frac{\partial^{2} w_{s t, i}}{\partial y \partial z}\right)^{2}\right)\right] d y d z .
\end{aligned}
$$


The total strain energy of the whole member with the transverse stiffeners therefore can be composed from Eqs. (16), (18) and (22):

$$
\Pi_{\mathrm{int}}=\Pi_{\mathrm{int}}^{S-V}+\Pi_{\mathrm{int}}^{\text {warp }}+\sum_{1}^{n_{s t}} \Pi_{\mathrm{int}, i}^{s t} .
$$

The total potential of the whole member is:

$$
\Pi=\Pi_{\mathrm{int}}+\Pi_{e x t} \text {. }
$$

In equilibrium the total potential is stationary, thus:

$$
\frac{\partial \Pi}{\partial c_{i}}=0, i=1, \ldots, k
$$

The critical load can be obtained from Eqs. (25). It is worth noting that if the number of parameters is larger than one (that is, if $k>1$ ), then the equations define a generalized eigen-value problem the eigen-values of which are the critical loads.

\subsection{Specific problem: I-section column with rectangular stiffeners}

The problem described in the previous Section is too complex to have an analytical solution in a general case. The difficulties are as follows: (i) the $w_{s t, i}(y, z)$ displacement function is sometimes hard to find, and (ii) the presence of the stiffeners has, in general, an important effect on the $\theta(x)$ function. In some specific cases, however, closedformed solution can be found to the problem. In the following Sections some specific cases are investigated: analytical solutions are presented and discussed. Also, the analytical solutions are compared to shell FEM solutions.

The specialty of the simplified problem is that the cross-section is a doubly-symmetrical I-section, and the stiffeners are rectangular plates, centrally positioned with respect to the cross-section. As Fig. 2(a) illustrates, the depth and width of the member cross-section is $h$ and $b$, respectively, interpreted as midline dimensions. The $i$-th stiffener plate has a thickness $t_{s t, i}$, its height and width are $h_{s t, i}$ and $b_{s t, i}$, respectively, and now it is assumed that $h_{s t, i} \leq h$ and $b_{s t, i} \leq b$.

The coordinate system defined in such a manner that its $\mathrm{O}$ origin would coincide the $\mathrm{C}$ centroid of the cross section, which is now identical to the shear center, too.

For the connection between the main member and the stiffeners, three cases will be considered. In the case of "flanges-only" connection the stiffener is connected to the flanges of the main member only. In the case of "webonly", the stiffener is connected to the web of the main

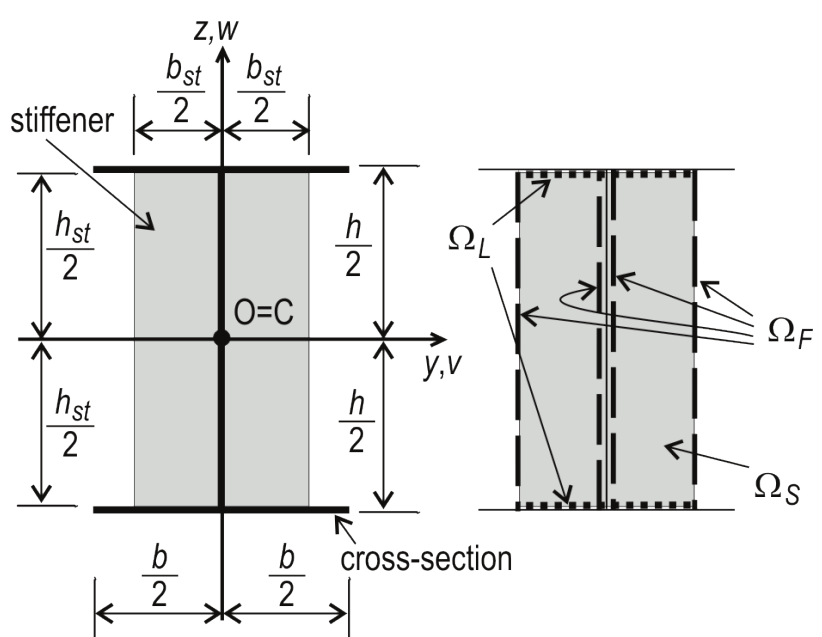

a)

b)

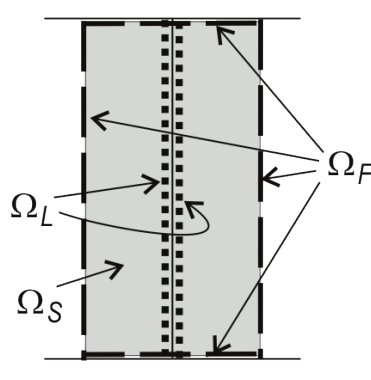

c)

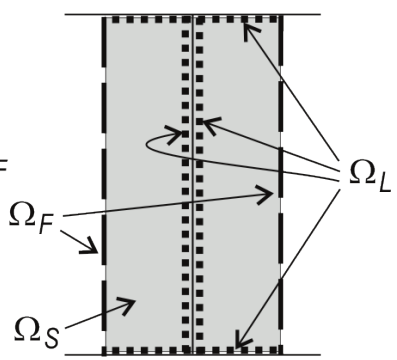

d)
Fig. 2 a) coordinates, dimensions, b) flanges-only connection, c) web-only connection, d) web-and-flanges connection

member only. In the case of "web-and-flanges" connection the stiffener is connected to both the web and flanges of the main member.

The most practical case is when the stiffener is connected both to the web and the flanges, still, the other two cases have been found to be useful. It will be shown (in Section 3.3) that for the "web-and-flanges" case there is no strong solution of the DE of the transverse plate if the cross-section of the main member is assumed to be rigid (which is the usual way to define any kind of global buckling). At the same time, if the stiffener is connected either to the web or the flanges only, exact analytical solution for the DE of the transverse plate is possible. Numerical studies will show (in Section 4.2) that the difference between the "flanges-only" and "web-and-flanges" cases is mostly small. That is why the approximate $w$ function for the "web-and-flanges" case will be assumed as the perturbation of the "flanges-only" case. To show the validity of the newly derived formulae, thus, "flanges-only" and "webonly" cases have also been considered to avoid the inaccuracy introduced by the unavoidable inaccuracy of the w function in the "web-and-flanges" case. 


\section{Analysis of stiffeners of I-section members}

\subsection{Stiffeners connected to flanges only}

The $w_{s t, i}$ displacement function of a stiffener plate is aimed to derive here, by assuming the displacement function in a polynomial form. The function should satisfy the differential equation of the plate, see Eq. (5), plus the boundary conditions, which are dependent on the stiffener-to-member connection.

The compatibility conditions at $z=h_{s t, i} / 2$ are as follows:

$w_{s t, i}=\theta_{s i}^{\prime} \frac{h_{s t, i}}{2} y$,

$\frac{\partial w_{s t, i}}{\partial z}=-\theta^{\prime}{ }_{s i} y$,

where: $\theta_{s i}^{\prime}$ is the first derivative (with respect to $x$ ) of the twisting rotation function at position $x=x_{s t, i}$.

The compatibility conditions at $z=-h_{s t, i} / 2$ are as follows:

$w_{s t, i}=-\theta_{s i}^{\prime} \frac{h_{s t, i}}{2} y$,

$\frac{\partial w_{s t, i}}{\partial z}=-\theta_{s i}^{\prime} y$.

The boundary condition for the free edges, i.e. at $y= \pm b_{s t, i} / 2$ are as follows:

$\frac{\partial^{2} w_{s t, i}}{\partial y^{2}}=0$

In this case the problem can be solved easily. The function that satisfies the Eq. (5) and the above boundary conditions is as follows:

$w_{s t, i}(y, z)=\theta_{s i}^{\prime} y z-\theta_{s i}^{\prime} 2 y\left(\frac{2}{h_{s t, i}{ }^{2}} z^{3}-\frac{z}{2}\right)$.

By using this function for the stiffener displacement, the strain energy can be calculated, by substituting Eq. (31) into Eqs. (19-22).

For the $i$-th stiffener the curvatures:

$\kappa_{s t, i, y y}=\frac{\partial^{2} w_{s t, i}}{\partial y^{2}}=0$

$\kappa_{s t, i, z z}=\frac{\partial^{2} w_{s t, i}}{\partial z^{2}}=-\theta_{s i}^{\prime} \frac{24}{h_{s t, i}{ }^{2}} y z$,

$\kappa_{s t, i, y z}=\frac{\partial^{2} w_{s t, i}}{\partial y \partial z}=-\theta_{s i}^{\prime}\left(\frac{12}{h_{s t, i}{ }^{2}} z^{2}-2\right)$.

Let us substitute Eqs. (32-34) into Eq. (22):

$$
\begin{aligned}
& \Pi_{\mathrm{int}, i}^{s t}=\int_{b_{s t, i}}^{\frac{b_{s t, i}}{2}} \int_{\frac{h_{s t, i}}{2}}^{\frac{h_{s, i}}{2}} \frac{D_{s t, i}}{2}\left(\theta_{s i}^{\prime}\right)^{2} \\
& {\left[\left(\frac{24}{h_{s t, i}{ }^{2}} y z\right)^{2}+2(1-v)\left(\frac{12}{h_{s t, i}{ }^{2}} z^{2}-2\right)^{2}\right] d y d z .}
\end{aligned}
$$

After performing the mathematical operations, the strain energy can be expressed as:

$\Pi_{\mathrm{int}, i}^{s t}=\left(\theta_{s i}^{\prime}\right)^{2} D_{s t, i} \frac{b_{s t, i}\left(10 b_{s t, i}{ }^{2}-9 h_{s t, i}{ }^{2} v+9 h_{s t, i}{ }^{2}\right)}{5 h_{s t, i}}$.

The above formula can be written in short as:

$\Pi_{\mathrm{int}, i}^{s t}=\left(\theta_{s i}^{\prime}\right)^{2} D_{s t, i} C_{s t, i}$,

with:

$C_{s t, i}=\frac{b_{s t, i}\left(10 b_{s t, i}{ }^{2}-9 h_{s t, i}{ }^{2} v+9 h_{s t, i}{ }^{2}\right)}{5 h_{s t, i}}$.

\subsection{Stiffeners connected to web only}

Let us consider the case when the stiffener is connected to the main member at the web only. Let us analyze the half of the stiffener, i.e., $y \geq 0$. The compatibility conditions at $y=0$ is as follows:

$w_{s t, i}=0$,

$\frac{\partial w_{s t, i}}{\partial y}=\theta_{s i}^{\prime} z$

The boundary condition at the free edges are as follows. At $z= \pm h_{s t, i} / 2$ :

$\frac{\partial^{2} w_{s t, i}}{\partial^{2} z}=0$

$$
\text { At } y= \pm b_{s t, i} / 2 \text { : }
$$

$\frac{\partial^{2} w_{s t, i}}{\partial^{2} y}=0$

It is easy to prove that the following two functions satisfy all the criteria:

$w_{s t, i 1}=\theta_{s i}^{\prime} y z$,

$w_{s t, i 2}=\theta_{s i}^{\prime}\left(y-\frac{3}{b_{s t, i}} y^{2}+\frac{2}{b_{s t, i}^{2}} y^{3}\right) z$.

The general form of plate displacement is searched as the linear combination of the two functions: 
$w_{s t, i}=c_{i} w_{s t, i 1}+\left(1-c_{i}\right) w_{s t, i 2}$

where $c_{i}$ is a scalar parameter. It can be found by minimizing the strain energy of the stiffener plate. From this minimization, the following formula can be derived:

$c_{i}=\frac{4 G b_{s t, i}{ }^{2}+5 E h_{s t, i}{ }^{2}}{24 G b_{s t, i}{ }^{2}+5 E h_{s t, i}^{2}}$.

The same analysis could be repeated for $y \leq 0$, which would lead to (essentially) the same results. The strain energy in the stiffener can finally be expressed by Eq. (37), but now the definition of $C_{s t, i}$ is different. Without showing the details, the relevant $C_{s t, i}$ is as follows:

$C_{s t, i}=\frac{h_{s t, i}{ }^{3}}{2 b s t_{i}}\left(c_{i}-1\right)^{2}+\frac{b_{s t, i} h_{s t, i}}{5}(1-v)\left(6 c_{i}^{2}-2 c_{i}+1\right)$.

\subsection{Stiffeners connected to web and flanges}

Let's analyze half of the plate, $y \geq 0$. The compatibility conditions are as follows. At $z=h_{s t, i} / 2$ :

$$
\begin{aligned}
& w_{s t, i}=\theta_{s i}^{\prime} \frac{h_{s t, i}}{2} y, \\
& \frac{\partial w_{s t, i}}{\partial z}=-\theta_{s i}^{\prime} y . \\
& \text { At } z=-h_{s t, i} / 2 . \\
& w_{s t, i}=-\theta_{s i}^{\prime} \frac{h_{s t, i}}{2} y, \\
& \frac{\partial w_{s t, i}}{\partial z}=-\theta_{s i}^{\prime} y . \\
& \text { At } y=0: \\
& w_{s t, i}=0, \\
& \frac{\partial w_{s t, i}}{\partial y}=\theta_{s i}^{\prime} z .
\end{aligned}
$$

The boundary condition for the free edges is as follows, i.e., at $b_{s t, i} / 2$ :

$\frac{\partial^{2} w_{s t, i}}{\partial^{2} y}=0$

The above-described boundary conditions lead to a discontinuity. The second derivative of $w_{s t, i}$ along the $z=h_{s t, i} / 2$ line, from Eq. (49) is:

$\frac{\partial^{2} w_{s t, i}}{\partial y \partial z}=-\theta_{s i}^{\prime}$.
On the other hand, the second derivative of $w_{s t, i}$ along the $y=0$ line, from Eq. (53):

$\frac{\partial^{2} w_{s t, i}}{\partial y \partial z}=\theta_{s i}^{\prime}$

In the intersection point of the $z=h_{s t, i} / 2$ line and the $y=0$ line, that is at the flange-to-web junction point, the second derivative of $w_{s t, i}$ is discontinuous (for any $\theta_{s i}^{\prime} \neq 0$ value). It is therefore not possible to find an exact, strong solution for the described plate problem. However, approximate solution is certainly possible. Here a simple approach is followed. It is easy to observe that the $w_{s t, i}$ function used for the flanges-only case satisfies almost all the criteria of the web-and-flanges case. The only exception is the first derivative along the $y=0$ line. Let us substitute Eq. (31) into Eq. (53):

$\frac{\partial w_{s t, i}}{\partial y}=\theta_{s i}^{\prime} z-\theta_{s i}^{\prime} 2\left(\frac{2}{h_{s t, i}^{2}} z^{3}-\frac{z}{2}\right) \neq \theta_{s i}^{\prime} z$.

To approximately satisfy condition Eq. (53) when $y=0$, but not to (or only to slightly) disturb the nature of Eq. (31) elsewhere, an additional function term is introduced into the displacement function as follows:

$$
w_{s t, i}(y, z)=\theta_{s i}^{\prime} y z-\theta_{s i}^{\prime} 2 y\left(\frac{2}{h_{s t, i}{ }^{2}} z^{3}-\frac{z}{2}\right)+f_{i}(y) \theta_{s i}^{\prime} 2\left(\frac{2}{h_{s t, i}{ }^{2}} z^{3}-\frac{z}{2}\right),
$$

where $f_{i}(y)$ function (i) should be zero at $y=0$, (ii) should have a unit first derivative at $y=0$, and (iii) should take non-zero values only in the vicinity of $y=0$, while should take zero values otherwise. These conditions are satisfied by the following function:

$f_{i}(y)=\left\{\begin{array}{cl}y-\frac{2}{\bar{b}_{s t, i}} y^{2}+\frac{1}{\bar{b}_{s t, i}{ }^{2}} y^{3} & \text { if } y \leq \bar{b}_{s t, i}, \\ 0 & \text { otherwise }\end{array}\right.$

where $\bar{b}_{s t, i}$ is a parameter that should somehow be assumed or approximated (According to the experiences from the numerical examples, $\bar{b}_{s t, i}$ can be assumed as $0.15 b_{s t, i}$ for the investigated problems).

Applying the same process as in the previous cases, the new internal potential energy of stiffener plates can be derived, resulting in a formulae identical to Eq. (37), but with a different $C_{s t, i}$ term. In this case the formula for the $C_{s t, i}$ is obtained as follows:

$C_{s t, i}=\frac{b_{s t, i}\left(10 b_{s t, i}{ }^{2}-9 h_{s t, i}{ }^{2} v+9 h_{s t, i}{ }^{2}\right)}{5 h s t_{i}}+\frac{8}{525} \frac{30 \bar{b}_{s t, i}{ }^{4}+14 \bar{b}_{s t, i}{ }^{2} h_{s t, i}{ }^{2}+5 h_{s t, i}{ }^{4}}{\bar{b}_{s t, i} h_{s t, i}}$ 


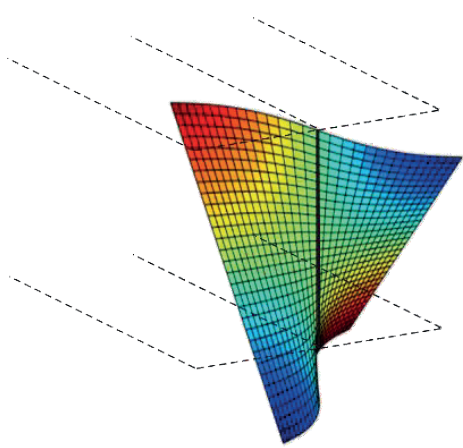

web-only

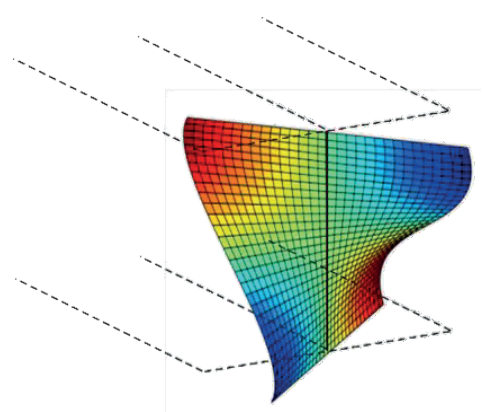

flanges-only

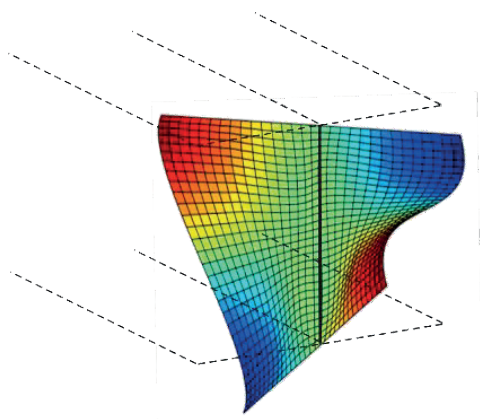

web-and-flanges

Fig. 3 Stiffener deformations

The $w_{s t, i}(y, z)$ displacement functions for the three cases are illustrated in Fig. 3. It is to observe that the deformation in the flanges-only and web-and-flanges cases are very similar, but certainly not identical, since some localized waviness around the web is visible in the latter case.

\section{Clamped-clamped I-column with stiffeners}

\subsection{Analytical solution}

The considered column is clamped-clamped, therefore the assumed longitudinal twisting displacement function is:

$\theta=\theta_{0} \frac{1}{2}\left[1-\cos \left(\frac{2 \pi x}{L}\right)\right]$

This is realistic if the stiffeners are relatively weak and regularly positioned (as we will see later). The first derivative of the function is:

$\frac{\partial \theta}{\partial x}=\frac{\pi}{L} \theta_{0} \sin \left(\frac{2 \pi}{L} x\right)$

By substituting Eq. (60) into Eqs. (13), (16) and (18), we obtain the energy function in the main member, as follows:

$$
\begin{aligned}
& \Pi_{e x t}=-F r_{0}{ }^{2} \frac{\pi^{2}}{4 L}\left(\theta_{0}^{2}\right), \\
& \Pi_{\mathrm{int}}^{S-V}=G I_{t} \frac{\pi}{12 L}\left(3 \pi \theta_{0}^{2}\right), \\
& \Pi_{\mathrm{int}}^{\text {warp }}=E I_{\omega} \frac{\pi^{3}}{12 L^{3}}\left(12 \pi \theta_{0}^{2}\right) .
\end{aligned}
$$

Let's analyze a stiffener plate at position $x=x_{s, i}$. Substitute $x=x_{s t, i}$ into Eq. (62):

$\theta_{s i}^{\prime}=\frac{\partial \theta}{\partial x \mid x=x_{s t, i,}}=\frac{\pi}{L} \theta_{0} \sin \left(\frac{2 \pi}{L} x_{s t, i}\right)$.

To get the strain energy in the stiffeners, we need to substitute Eq. (66) into Eq. (37). For one stiffener:
$\Pi_{\mathrm{int}, i}^{s t}=\frac{\pi^{2}}{L^{2}}\left(\theta_{0}\right)^{2} \sin ^{2}\left(\frac{2 \pi}{L} x_{s t, i}\right) D_{i} C_{s t, i}$.

From Eq. (67) it is obvious that $\Pi_{i n t, i}^{s t}$ is dependent on the position of the stiffener, i.e., on $x_{s t, i}$. It is also dependent on how it is connected to the main member, reflected in the $C_{s t, i}$ term. To obtain the total potential energy function, we need to summarize the above energy terms, as in Eq. (23):

$\Pi=G I_{t} \frac{\pi}{12 L} 3 \pi \theta_{0}^{2}+E I_{\omega} \frac{\pi^{3}}{12 L^{3}} 12 \pi \theta_{0}^{2}$

$+\frac{\pi^{2}}{L^{2}} \theta_{0}^{2} D_{s t, i} C_{s t, i} \sum_{1}^{n_{s t}} \sin ^{2}\left(\frac{2 \pi}{L} x_{s t, i}\right)-F r_{0}^{2} \frac{\pi^{2}}{4 L} \theta_{0}^{2}$.

The minimum of the potential energy can be found where its first derivative is zero:

$$
\begin{aligned}
& \frac{\partial \Pi}{\partial \theta_{0}}=G I_{t} \frac{\pi}{2 L} \pi \theta_{0}+E I_{\omega} \frac{\pi^{3}}{L^{3}} 2 \pi \theta_{0} \\
& +D_{s t, i} C_{s t, i} \frac{\pi^{2}}{L^{2}} 2 \theta_{0} \sum_{1}^{n_{s t}} \sin ^{2}\left(\frac{2 \pi}{L} x_{s t, i}\right)-F r_{0}^{2} \frac{\pi^{2}}{2 L} \theta_{0}=0 .
\end{aligned}
$$

From Eq. (69) the critical force can be expressed as follows:

$$
F=\frac{1}{r_{0}^{2}}\left(G I_{t}+4 E I_{\omega} \frac{\pi^{2}}{L^{2}}+\frac{4}{L} \sum_{1}^{n_{s t}} D_{s t, i} C_{s t, i} \sin ^{2}\left(\frac{2 \pi}{L} x_{s t, i}\right)\right) .
$$

Eq. (70) indicates that there is an additional term in the critical force formula due to the presence of the stiffeners. The effect of the stiffeners is reflected in and only in this third term. This additional term can be interpreted as a weighted sum, in which the $4 D_{s t, i} C_{s t, i} / L$ term is due to the deformation (hence: accumulated strain energy) in one stiffener, while the $\sin \left(\frac{2 \pi}{L} x_{s, i}\right)^{2}$ terms are the weights. These weights are related to the first derivative of the twist function, since $\sum_{i}^{n_{s t}} \sin ^{2}\left(\frac{2 \pi}{L} x_{s t, i}\right)=\frac{L^{2}}{\pi^{2}} \frac{1}{\theta_{0}} \sum_{1}^{n_{s t}}\left(\theta_{s i}^{\prime}\right)^{2}$. Thus, the larger the value of the first derivative of the twisting rotation 
function, the more effective the stiffener against torsion. It is also to highlight that (a) the Saint-Venant torsional term is independent of the length, (b) the warping term is inversely proportional to $L^{2}$, and (c) the term due to the stiffeners is inversely proportional to $L$. Thus, the effect of stiffeners cannot be reasonably represented by neither a modified $I_{t}$, nor a modified $I_{\omega}$.

It is interesting to mention that if the stiffeners are identical then Eq. (70) can be written as:

$$
F=\frac{1}{r_{0}^{2}}\left(G I_{t}+4 E I_{\omega} \frac{\pi^{2}}{L^{2}}+\frac{4}{L} D_{s t} C_{s t} \sum_{1}^{n_{s t}} \sin ^{2}\left(\frac{2 \pi}{L} x_{s t, i}\right)\right) .
$$

Furthermore, if the stiffeners are equally spaced (i.e., in the case of 1 stiffener it is in the middle of the member, in the case of 2 stiffeners they are in the third points of the member, etc.), then:

$$
\sum_{1}^{n_{s t}} \sin ^{2}\left(\frac{2 \pi}{L} x_{s t, i}\right)=\sum_{1}^{n} \sin ^{2}\left(\frac{2 \pi}{n_{s t}+1} i\right),
$$

which finally leads to:

$$
\sum_{1}^{n_{s t}} \sin ^{2}\left(\frac{2 \pi}{n_{s t}+1} i\right)=\frac{n_{s t}+1}{2} \text { if } n_{s t}>1 \text {. }
$$

This means that the third term in the critical force formula is linearly dependent on the number of (equally spaced, identical) stiffeners.

\subsection{Example \#1: one single stiffener}

In this example some elementary problems are solved, in order to demonstrate the effect of the various parameters of the stiffeners, and also to validate the analytical solution against finite element solutions. A clamped-clamped I-section column member is considered with one single stiffener. The critical load to pure torsional buckling is calculated by the above presented analytical approach/formulae. Moreover, the critical loads are calculated by shell finite element analysis, using Ansys [15].

The column length varies, but relatively long members are considered in order to keep the critical force/stress at a low value, in order to avoid the local buckling from the first buckling modes from the FE analysis. The cross-section is similar to an HEA300 hot-rolled steel profile. More specifically the cross-section depth is $h=300 \mathrm{~mm}$, the width is $b=300 \mathrm{~mm}$, the flange thickness is $t_{f}=20.5 \mathrm{~mm}$, the web thickness is $t_{w}=11.5 \mathrm{~mm}$. (The depth and width values are interpreted for the midline of the cross-section.) The material is isotropic linearly elastic steel, with $E=210000 \mathrm{MPa}$ and $v=0.3$.
The stiffener width and height is equal to the width and depth of the cross-section. The $t_{s t}$ stiffener thickness varies between $0.5 t_{w}$ and $5 t_{w}$. Its material is identical to that of the main member. The position of the stiffener varies along the length.

Two concentric axial compressive forces are applied at the member ends, equal in magnitude but opposite in direction. The forces are put to the member as distributed loads uniformly distributed over the cross-section.

In the shell FE model SHELL63 elements are used, since these elements are based on the Kirchhoff-Love thin plate theory (just like the analytical solution). The sizes of the shell elements were kept approx. $50 \mathrm{~mm}$, which might seem to be a rough discretization, but mesh sensitivity studies proved the appropriateness of this element size. (It is to note that the analyzed phenomenon is global buckling, without significant localized deformations, that is why fine discretization is not necessary.)

In this example the member ends are clamped. In the shell FEM model the clamped supports were realized by rigid constraints (i.e., CERIG command in Ansys). All the nodes of the end-section are linked to a master node by rigid constraints, by defining the constraints so that all degrees of freedom of the nodes are linked to the master node. Then the master node is supported as usual at a clamped support in a beam model (i.e. preventing rotations and transverse translations, but allowing longitudinal translation).

In Table 1 the critical stresses are given in $\mathrm{N} / \mathrm{mm}^{2}$, for the three types of stiffener-to-member connection. The presented results belong to $L=8 \mathrm{~m}$, stiffener position $x_{s}=2000 \mathrm{~mm}$, and $t_{s t}$ is either $t_{w}$ and $2 t_{w}$. Both analytical and FEM results are shown. The more important observations are as follows

The analytical and shell FEM results are not identical, showing a few percent difference. This observation is in line with previous experiences, since the analytical solution is based on a beam-model, while the FEM solution is on a shell-model. This question is discussed in detail in e.g., [16], where the sources of the small differences are identified. Nevertheless, as far as the effect of the stiffener is concerned, the shell FEM and analytical solutions show very similar tendencies.

The increment caused by the stiffener is greatly dependent on the stiffener-to-member connection. Web-only connection induces a very moderate increment, in the order of $1 \%$. Flange-only connection is much more efficient, the increment is in the order of $10 \%$. If connection 
Table 1 Critical stresses with one stiffener, $L=8 \mathrm{~m}, x_{s}=2 \mathrm{~m}$

\begin{tabular}{lcccc}
\hline & & no stiffener & web-only & flanges-only \\
\hline$t_{s t}=t_{w}$ & analytical & 1064.3 & 1065.5 & 1075.2 \\
$t_{s t}=t_{w}$ & FEM & 1051.9 & 1052.5 & 1060.3 \\
$t_{s t}=2 t_{w}$ & analytical & 1064.3 & 1073.3 & 1060.7 \\
$t_{s t}=2 t_{w}$ & FEM & 1051.9 & 1055.3 & 1161.2 \\
\hline
\end{tabular}

is provided both at the web and flanges, the increment is the largest, approximately the sum of the increments caused by the web-only and flanges-only connections separately.

Obviously, the increment of the critical load due to the stiffener is largely influenced by the thickness of the stiffener: the thicker the stiffener, the larger the increment. However, it can also be observed that the difference of the FEM and analytical critical values is getting larger as the stiffener thickness increases. This tendency will be observable throughout all the other examples in the paper, and can be explained by two factors. One factor is that in the analytical solution the effect of the stiffeners on the longitudinal displacement function of the member (i.e., the theta twisting function) is not considered, whilst such effect obviously exists and naturally included in the FEM calculations (as will clearly be demonstrated in some examples). The other factor is that in the analytical solution the cross-sections are rigid and the plane element are free from in-plane shear (due to the beammodel approach), whilst in the shell FEM model such conditions are not enforced. For longer members the pure-torsional buckled shape from the shell FEM is very similar to that assumed in the analytical solution (that is why the critical values from FEM and analytical solutions are very similar), but not identical. These differences of the deformations are increased if stiffeners are added. (More discussion of this question requires modal buckling analysis, which will be presented by the authors in another paper.)

On the basis of the observations from Table 1, in the following examples mostly the flanges-only cases will be discussed, since for this type of connection the stiffener displacement function $w_{s t}$ is precisely known and the effect of the stiffener is pronounced.
In Table 2 the critical stresses are given, for various stiffener positions (measured from one end of the member), calculated for $L=8 \mathrm{~m}$ and for flanges-only connection. Both analytical and FEM results are shown for two $t_{s t}$ values. The values in the table are the increments (in $\mathrm{N} / \mathrm{mm}^{2}$ ) caused by the stiffener, with respect to the critical stresses without the stiffener $\left(1064.1 \mathrm{~N} / \mathrm{mm}^{2}\right.$ and $1052.4 \mathrm{~N} / \mathrm{mm}^{2}$ from the analytical and FEM calculations, respectively, see Table 1).

It can be observed that the tendencies of the analytical and FEM results are the same, in the case of the thinner stiffener even the numerical values are fairly similar. According to the analytical solution, see Eq. (70), the increment of the critical force due to the stiffeners is:

$\Delta F^{s t}=\frac{4}{L} \sum_{1}^{n_{s t}} D_{s t, i} C_{s t, i} \sin ^{2}\left(\frac{2 \pi}{L} x_{s t, i}\right)$,

which is, since now there is one single stiffener, simplified to:

$\Delta F^{s t}=\frac{4}{L} D_{s t} C_{s t} \sin ^{2}\left(\frac{2 \pi}{L} x_{s t}\right)$

The effect of the stiffener position is included only in the sinusoidal term, so the increments in any row of Table 2 should be proportional to the corresponding $(\sin )^{2}$ values as follows:

$\sin ^{2}\left(\frac{\pi}{4}\right): \sin ^{2}\left(\frac{2 \pi}{4}\right): \sin ^{2}\left(\frac{3 \pi}{4}\right): \sin ^{2}\left(\frac{4 \pi}{4}\right) \Rightarrow \frac{1}{2}: 1: \frac{1}{2}: 0$.

Indeed, the increments in Table 2 follow precisely this pattern in the case of the analytical calculation, but also practically precisely in the case of the shell FEM calculation.

In Table 3 the effect of the member length is demonstrated. The presented numerical values are calculated with $t_{s t}=t_{w}$ and $x_{s}=0.25 \mathrm{~L}$. It is to note that the considered

Table 2 Critical stress increment due to one stiffener, $L=8 \mathrm{~m}$, flanges-only connection

\begin{tabular}{lcccc}
\hline & & $1000 \mathrm{~mm}$ & $2000 \mathrm{~mm}$ & $3000 \mathrm{~mm}$ \\
\hline$t_{s t}=t_{w}$ & analytical & 5.4 & 10.9 & 5.4 \\
$t_{s t}=t_{w}$ & FEM & 4.2 & 8.4 & 4.2 \\
$t_{s t}=2 t_{w}$ & analytical & 43.4 & 86.8 & 0.0 \\
$t_{s t}=2 t_{w}$ & FEM & 21.1 & 42.7 & 43.4 \\
\hline
\end{tabular}


Table 3 Critical stresses with one stiffener, $t_{s t}=t_{w}, x_{s}=0.25 L$, flanges-only connection

\begin{tabular}{lcccccc}
\hline & & $6 \mathrm{~m}$ & $8 \mathrm{~m}$ & $10 \mathrm{~m}$ & $12 \mathrm{~m}$ & $16 \mathrm{~m}$ \\
\hline no stiffener & analytical & 1594.2 & 1064.3 & 819.1 & 685.9 & 553.4 \\
no stiffener & FEM & 1561.3 & 1052.4 & 812.5 & 681.5 & 551.0 \\
with stiffener & analytical & 1608.7 & 1075.2 & 827.8 & 693.1 & 558.8 \\
with stiffener & FEM & 1573.0 & 1061.0 & 819.3 & 687.2 & 555.4 \\
\hline
\end{tabular}

Table 4 Critical stress increments due to one stiffener, $L=8 \mathrm{~m}, x_{\mathrm{s}}=2 \mathrm{~m}$, flanges-only connection

\begin{tabular}{lccccc}
\hline & $t_{s t} / t_{w}=0.5$ & $t_{s t} / t_{w}=1$ & $t_{s t} / t_{w}=1.5$ & $t_{s t} / t_{w}=2$ & $t_{s t} / t_{w}=5$ \\
\hline analytical & 1.357 & 10.85 & 36.63 & 86.82 & 1357 \\
FEM & 0.958 & 8.447 & 23.06 & 41.53 & 244.3 \\
\hline
\end{tabular}

members are fairly long, which was necessary to avoid the dominance of local buckling. It is also worth noting that the FEM and analytical results are getting closer as the member length increases. According to Eq. (74) the increment should be inversely proportional to the member length. Thus, if the increments are plotted in the function of the member length, they should follow a hyperbola. This is obviously satisfied by the analytical results, but also very well approximated by the shell FEM results, as shown in Fig. 4, top. If they are plotted in a doubly logarithmic scale, they should lay on a straight line with a $-45 \mathrm{deg}$
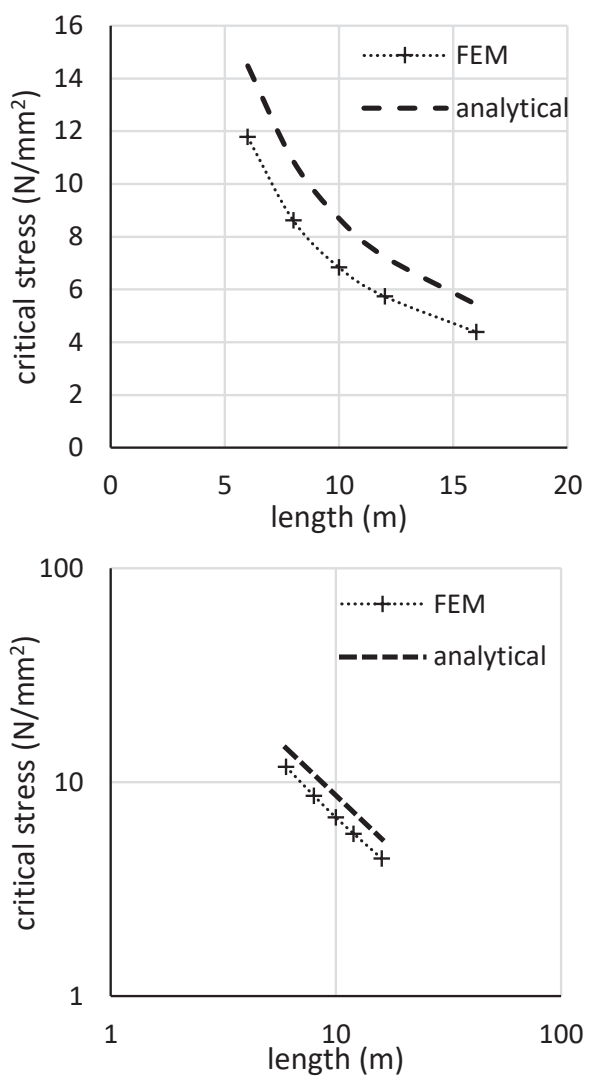

Fig. 4 Critical stress increments due to one stiffener, $t_{s t}=t_{w}, x_{s}=0.25 \mathrm{~L}$, flanges-only connection inclination. This is shown in Fig. 4, bottom. Though there is a systematic difference between the FEM and the analytical results, the tendencies of the FEM results perfectly follow the ones predicted by the analytical formula.

In Table 4 the effect the stiffener thickness is illustrated.

In Table 4 the stress increments are given for various values of $t_{s t}$, for $L=8 \mathrm{~m}$ and $x_{s}=2 \mathrm{~m}$. According to the analytical solution, the stiffener thickness is included in (and only in) the $D$ plate stiffness, therefore the increment is proportional to $t_{s t}{ }^{3}$. This is precisely satisfied by the analytical results, but not satisfied by the shell FEM results, even though the increments are significantly increasing with the increase of the stiffener thickness.

As already mentioned above, the difference between the shell FEM and analytical results is due to the small differences between the shell-models and beam-models, and also due to the effect of the stiffeners on the longitudinal displacement distribution. This latter one can clearly be observed by the buckling shapes, see Fig. 5 . While in the case of a thin stiffener the longitudinal displacement (i.e., twist) distribution closely follows Eq. (61), if the stiffener is thick, the longitudinal displacement distribution of the buckled shape is distinctly different: though the deformed shape of the member is globally similar, it also has localized deformations around the thick stiffener, most visible in the flanges, see the right part of Fig. 5.

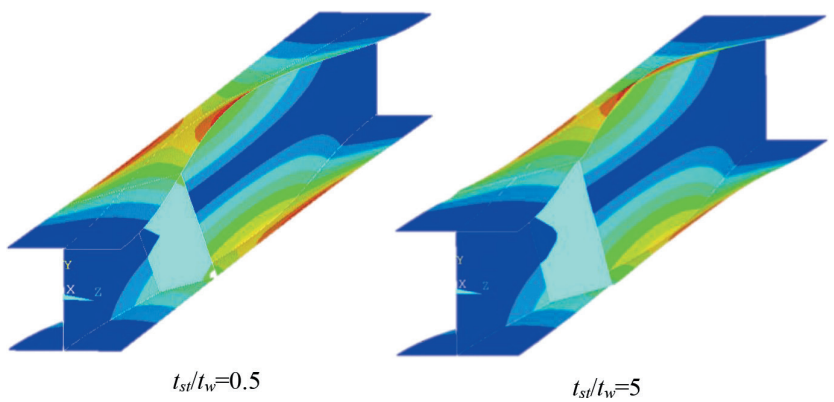

Fig. 5 Buckling shapes, $L=8 \mathrm{~m}, x_{s}=2 \mathrm{~m}$, flanges-only connection 


\subsection{Example \#2: multiple stiffeners}

In this Example \#2 the problem is essentially identical to that of Example \#1, but there are multiple stiffeners. The member length is $L=8 \mathrm{~m}$. The stiffeners are equally spaced.

The plots in Fig. 6 show that the critical load (or load increment) is linearly changing with the number of stiffeners, as predicted by the analytical formula, see Eq. (73), at least if there are more than one stiffener. The linearly increasing tendency is clearly observable from the shell FEM results, too. (It is to note that in certain cases it is not possible to find a pure torsional mode in the FEM solution, due to the large number of buckling modes with lower critical load values. This is the situation, for example, with the 8-m-long column having more than 10 stiffeners with $t_{s t}=5 t_{w}$, see Fig. 6.) The plots also demonstrate what has already been observed: the thicker the stiffener, the larger the differences between the shell FEM and analytical results. In Fig. 7 buckled shapes are shown from shell FEM: it can be observed that thick stiffeners generate waviness in the flanges.
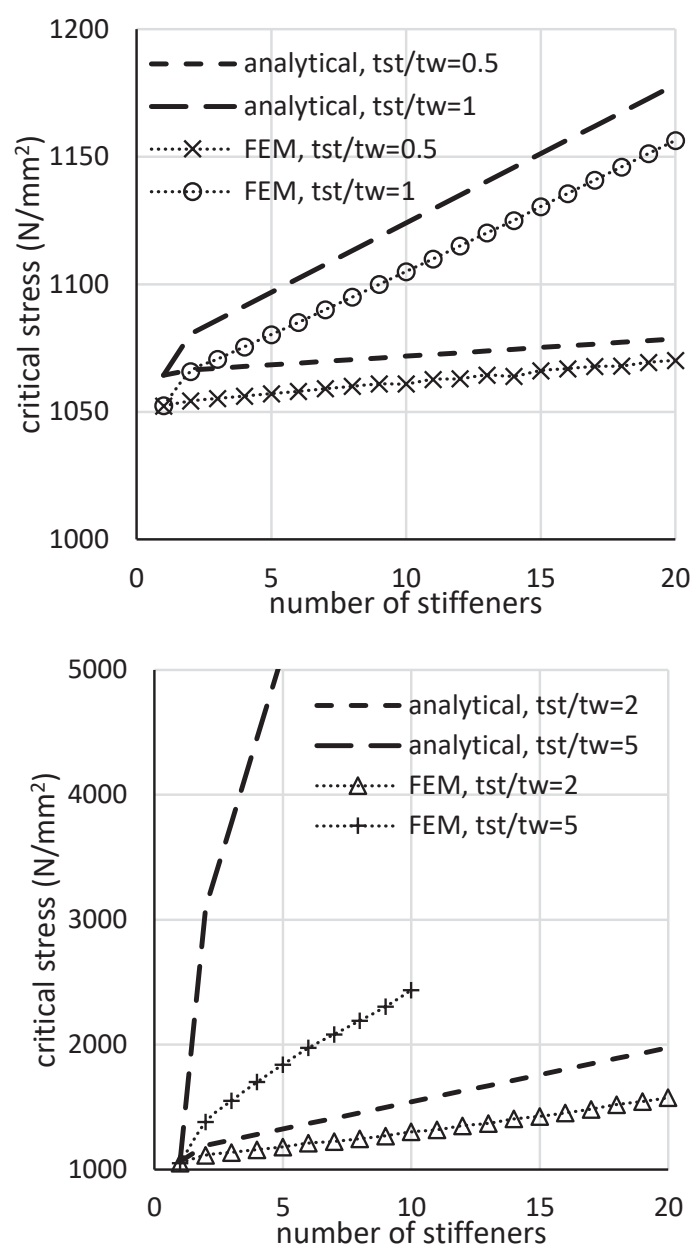

Fig. 6 Critical stresses in the function of the number of stiffeners, $L=8 \mathrm{~m}$

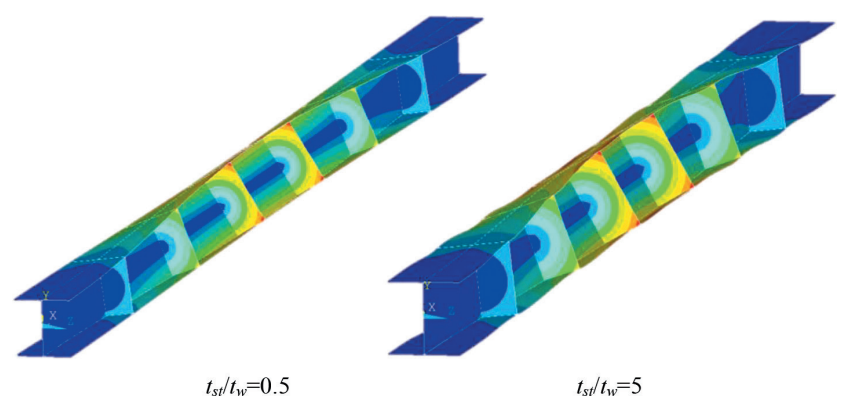

Fig. 7 Buckling shapes, $\mathrm{L}=8 \mathrm{~m}, 6$ stiffeners

\section{Pinned-pinned I-column with end-plates \\ 5.1 Analytical solution}

In Section 5 an I-section column member is studied again, similar to the one in Section 4, but with two differences: the member is pinned-pinned, and end-plates are assumed at the member ends. Thus, there are two stiffeners, at and. The primary goal here is to explicitly demonstrate the effect of the stiffeners on the longitudinal displacement function. It is very logical to assume that (i) if the endplates are very thin, then the behavior of the column will approximate a classic Euler column, and (ii) if the endplates are very thick, then the behavior of the member will be similar to that of a clamped-clamped column. In the first case the displacement function (for the first buckling mode with the lowest critical force) would be a half sinewave, while on the second case the displacement function would be a cosine function, just as in Eq. (61). Accordingly, the twisting displacement function is assumed as a linear combination of these two functions, as follows:

$\theta=\theta_{0,1} \sin \left(\frac{\pi x}{L}\right)+\theta_{0,2} \frac{1}{2}\left[1-\cos \left(\frac{2 \pi x}{L}\right)\right]$.

To obtain the potential energy in the main member, we need to substitute Eq. (77) into Eqs. (13), (16) and (18). The external potential is as follows:

$$
\begin{aligned}
& \Pi_{e x t}=-\frac{F}{2} r_{0}^{2} \int_{0}^{L}\left(\frac{\pi}{L} \theta_{0,1} \cos \left(\frac{\pi}{L} x\right)+\frac{\pi}{L} \theta_{0,2} \sin \left(\frac{2 \pi}{L} x\right)\right)^{2} d x, \\
& \Pi_{e x t}=-F r_{0}^{2} \frac{\pi}{12 L}\left(3 \pi \theta_{0,1}^{2}+16 \theta_{0,1} \theta_{0,2}+3 \pi \theta_{0,2}{ }^{2}\right) .
\end{aligned}
$$

The strain energy due to Saint-Venant strains and stresses:

$$
\begin{aligned}
& \Pi_{\mathrm{int}}^{S-V}=\frac{1}{2} G I_{t} \int\left(\frac{\partial \theta}{\partial x}\right)^{2} d x \\
& =\frac{1}{2} G I_{t} \int_{0}^{L}\left(\frac{\pi}{L} \theta_{0,1} \cos \left(\frac{\pi}{L} x\right)+\frac{\pi}{L} \theta_{0,2} \sin \left(\frac{2 \pi}{L} x\right)\right)^{2} d x, \\
& \Pi_{\mathrm{int}}^{S-V}=G I_{t} \frac{\pi}{12 L}\left(3 \pi \theta_{0,1}{ }^{2}+16 \theta_{0,1} \theta_{0,2}+3 \pi \theta_{0,2}{ }^{2}\right) .
\end{aligned}
$$


The strain energy due to warping strains and stresses:

$$
\begin{aligned}
& \Pi_{\mathrm{int}}^{\text {warp }}=\frac{1}{2} E I_{\omega} \int\left(\frac{\partial^{2} \theta}{\partial x^{2}}\right)^{2} d x \\
& =\frac{E I_{\omega}}{2} \int_{0}^{L}\left(-\frac{\pi^{2}}{L^{2}} \theta_{0,1} \sin \left(\frac{\pi}{L} x\right)+\frac{2 \pi^{2}}{L^{2}} \theta_{0,2} \cos \left(\frac{2 \pi}{L} x\right)\right)^{2} d x, \\
& \Pi_{\mathrm{int}}^{\text {warp }}=\frac{E I_{\omega} \pi^{3}}{12 L^{3}}\left(3 \pi \theta_{0,1}{ }^{2}+16 \theta_{0,1} \theta_{0,2}+12 \pi \theta_{0,2}{ }^{2}\right) .
\end{aligned}
$$

To obtain the strain energy accumulated in the endplates, we can apply Eq. (37).

$$
\Pi_{\text {int }}^{s t}=\left(\theta_{s 1}^{\prime}\right)^{2} D_{s t, 1} C_{s t, 1}+\left(\theta_{s 1}^{\prime}\right)^{2} D_{s t, 2} C_{s t, 2} \text {. }
$$

$C_{s t, 1}$ and $C_{s t, 2}$ are dependent on the plate parameters and on the stiffener-to-member connection, as we have seen in Section 4. $D_{s t, 1}$ and $D_{s t, 2}$ are dependent on the material and the plate thickness. For the actual case we can assume identical end-plates. Moreover, for the actual case $\theta_{s i}^{\prime}$, i.e. the rate of twisting rotation at the stiffener positions $x=x_{s t, i}$, can be calculated as follows:

$\theta_{s i}^{\prime}=\frac{\pi}{L} \theta_{0,1} \cos \left(\frac{\pi}{L} x_{s t, i}\right)+\frac{\pi}{L} \theta_{0,2} \sin \left(\frac{2 \pi}{L} x_{s t, i}\right)$.

For the end-plates this reads as:

$\theta_{s 1}^{\prime}=\frac{\pi}{L} \theta_{0,1} \quad \theta_{s 2}^{\prime}=-\frac{\pi}{L} \theta_{0,1}$

Thus, the strain energy in the two end-plates are identical. E.g., for the first end-plate:

$\Pi_{\text {int, } 1}^{s t}=\left(\theta_{s 1}^{\prime}\right)^{2} D_{s t} C_{s t}=\frac{\pi^{2}}{L^{2}} \theta_{0,1}^{2} D_{s t} C_{s t}$.

The total potential energy function is the sum of the above energy terms, expressed by two displacement parameters $\theta_{0,1}$ and $\theta_{0,2}$.

$$
\begin{aligned}
& \Pi=G I_{t} \frac{\pi}{12 L}\left(3 \pi \theta_{0,1}{ }^{2}+16 \theta_{0,1} \theta_{0,2}+3 \pi \theta_{0,2}{ }^{2}\right) \\
& +E I_{\omega} \frac{\pi^{3}}{12 L^{3}}\left(3 \pi \theta_{0,1}{ }^{2}+16 \theta_{0,1} \theta_{0,2}+12 \pi \theta_{0,2}{ }^{2}\right) \\
& +2 \frac{\pi^{2}}{L^{2}} \theta_{0,1}{ }^{2} D_{s t} C_{s t}-F r_{0}{ }^{2} \frac{\pi}{12 L}\left(3 \pi \theta_{0,1}{ }^{2}+16 \theta_{0,1} \theta_{0,2}+3 \pi \theta_{0,2}{ }^{2}\right) .
\end{aligned}
$$

In equilibrium the total potential is stationary, therefore:

$$
\frac{\partial \Pi}{\partial \theta_{0,1}}=0, \frac{\partial \Pi}{\partial \theta_{0,2}}=0,
$$

which leads to two equations as follows:
$G I_{t}\left(3 \pi \theta_{0,1}+8 \theta_{0,2}\right)+E I_{\omega} \frac{\pi^{2}}{L^{2}}\left(3 \pi \theta_{0,1}+8 \theta_{0,2}\right)$

$+\frac{24 \pi}{L} D_{s t} C_{s t} \theta_{0,1}-F r_{0}^{2}\left(3 \pi \theta_{0,1}+8 \theta_{0,2}\right)=0$,

or:

$G_{t}\left(8 \theta_{0,1}+3 \pi \theta_{0,2}\right)+E I_{\omega} \frac{\pi^{2}}{L^{2}}\left(8 \theta_{0,1}+12 \pi \theta_{0,2}\right)$

$-F r_{0}^{2}\left(8 \theta_{0,1}+3 \pi \theta_{0,2}\right)=0$.

In matrix form:

$\left[\begin{array}{cc}3 \pi\left(F_{1}-F\right) & 8\left(F_{12}-F\right) \\ 8\left(F_{12}-F\right) & 3 \pi\left(F_{2}-F\right)\end{array}\right]\left[\begin{array}{l}\theta_{0,1} \\ \theta_{0,2}\end{array}\right]=\left[\begin{array}{l}0 \\ 0\end{array}\right]$,

with:

$$
\begin{aligned}
& F_{1}=\frac{1}{r_{0}^{2}}\left(G I_{t}+E I_{\omega} \frac{\pi^{2}}{L^{2}}+\frac{8 D_{s t} C_{s t}}{L}\right), \\
& F_{12}=\frac{1}{r_{0}^{2}}\left(G I_{t}+E I_{\omega} \frac{\pi^{2}}{L^{2}}\right), \\
& F_{2}=\frac{1}{r_{0}^{2}}\left(G I_{t}+4 E I_{\omega} \frac{\pi^{2}}{L^{2}}\right),
\end{aligned}
$$

where: $F_{1}$ is the critical force that belongs to a displacement function $\theta_{1}=\theta_{0,1} \sin (\pi x / L)$, and $F_{2}$ is the critical force that belongs to a displacement function $\theta_{2}=\theta_{0,2} 1 / 2[1-\cos (2 \pi x / L)]$. In this specific example $F_{12}$ is equal to the critical force of a pinned-pinned column without stiffeners.

Eq. (92) can be interpreted as an eigen-value problem as:

$\mathbf{A} \boldsymbol{\theta}-F \boldsymbol{\theta}=\mathbf{0}$,

with

$\mathbf{A}=\left[\begin{array}{cc}3 \pi F_{1} & 8 F_{12} \\ 8 F_{12} & 3 \pi F_{2}\end{array}\right], \quad \boldsymbol{\theta}=\left[\begin{array}{c}\theta_{0,1} \\ \theta_{0,2}\end{array}\right]$.

If $\mathbf{A}$ is positive definite, then the above problem has two real and positive eigen-values, i.e., two real and positive $F$ values. Indeed, it can be proved that $\mathbf{A}$ is positive definite, hence we can always expect two positive critical forces. To actually have the solutions, we can interpret Eq. (92) as a homogeneous linear equation system, the non-trivial solution of which exists if the coefficient matrix is singular. Thus:

$\left|\begin{array}{cc}3 \pi\left(F_{1}-F\right) & 8\left(F_{12}-F\right) \\ 8\left(F_{12}-F\right) & 3 \pi\left(F_{2}-F\right)\end{array}\right|=0$,

which leads to a quadratic equation in $\mathrm{F}$, as follows:

$9 \pi^{2}\left(F-F_{1}\right)\left(F-F_{2}\right)-64\left(F-F_{12}\right)^{2}=0$. 
From the above quadratic equation the critical force can be expressed in closed form. There are two positive solutions, from practical point of view the smaller value is the more important one.

Lower and upper bounds for the critical force can be found. $F_{12}$ is a critical force to a member (without endplates) when the end cross-section can freely warp. Moreover, $F_{2}$ is a critical force to a member when the end cross-sections do not warp at all. If the member ends are pinned but end-plates are present, the end-section warping is partially (i.e., elastically) restrained, therefore the (lowest) critical value must be between $F_{12}$ and $F_{2}$. Moreover, the second term of Eq. (97) is positive, thus, its first term must be positive, too. This can be satisfied if either both $\left(F-F_{1}\right)$ and $\left(F-F_{2}\right)$ are positive or both are negative. Since $F$ must be smaller than $F_{2}$, both $\left(F-F_{1}\right)$ and $\left(F-F_{2}\right)$ must be negative. This finally means that

$$
F_{12}<F<\min \left(F_{1}, F_{2}\right) \text {. }
$$

Thus, lower and upper bounds are found. By looking at the formulae for $F_{1}$ and $F_{2}$, it is expected that $F_{1}$ is the upper bound in the case of thin end-plates, while $F_{2}$ is the upper bound for thicker end-plates.

\subsection{Example \#3: pinned-pinned member, two end-plates} In this Example \#3 the problem is similar to that of Example \#1: the cross-section and material are the same, but now the end supports are pinned and there are endplates at both member ends (and no further stiffeners). In Fig. 8 the critical stresses are plotted (for two member lengths) in the function of the end-plate thickness.

If the end-plate is thin, the FEM and the analytical solutions are practically identical, which solutions are practically identical to the critical stress of a pinned-pinned

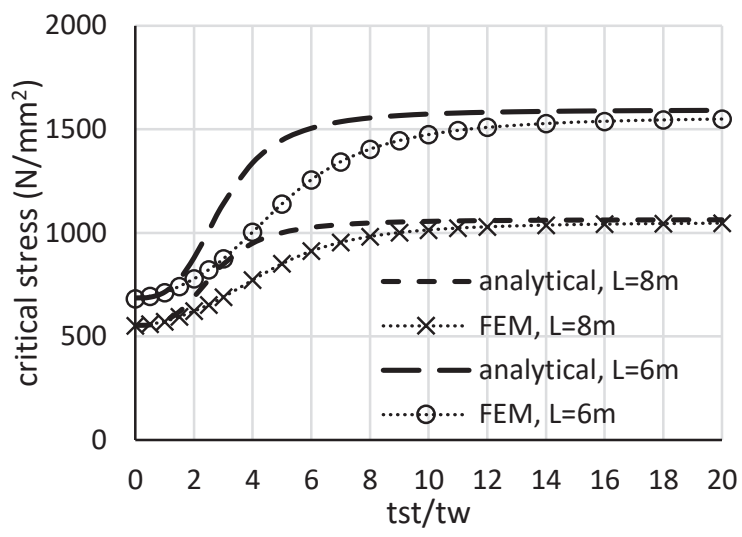

Fig. 8 Critical stresses, pinned member with two end-plates, FEM and analytical solutions member without end-plates. Again, if the end-plate is sufficiently thick, the FEM and the analytical solutions are nearly identical, which solutions are practically identical to the critical stress of a clamped-clamped member (with or without end-plates). The plot suggests that the necessary thickness to have a solution close to the clamped-clamped case is dependent on the column length: the shorter the column, the thicker end-plate is necessary. In any case, a fairly thick end-plate is necessary: even if the column is long (e.g., $8 \mathrm{~m}$ ), $t_{s t} / t_{w}$ should be around 10, i.e., the necessary end-plate thickness is more than $100 \mathrm{~mm}$. (It is to note that the precision of the applied thin plate theory is questionable for such thick plates.) Characteristic buckled shapes are shown in Fig. 9 for two thickness values, proving that the buckled shape transforms from the pinnedpinned to clamped-clamped as the thickness increases.

From Fig. 8 it is also clear that in the case of thick and moderately thick end-plates the difference between the shell FEM and the analytical prediction of the critical load is significant. As already stated above, the difference is caused by the small differences between the shell-modelbased and beam-model-based deformations, i.e., in-plane shear strains, plate-like deformations of the web and flanges of the member, etc., which are allowed in the shell model, but excluded from the beam model. Also, whilst in the analytical solution the longitudinal distribution of the twisting rotations is strictly prescribed by Eq (77), the shell FEM model is allowed to deviate from this strict prescription.

In Section 5.1 it has been shown that the critical load is bounded. This is illustrated in Fig. 10, where the relative critical load increments are plotted from the analytical solution, together with upper bounds $F_{1}$ and $F_{2}$, where the relative values are related to $F_{12}$. For example, the relative increment of $F_{1}$ is interpreted as $\left(F_{1}-F_{12}\right) / F_{12}$, and so on. (It is to note that in this plot the lower bound is the zero line, since the increment of $F_{12}$ with respect to $F_{12}$ is obviously zero.) Fig. 10 also demonstrates that

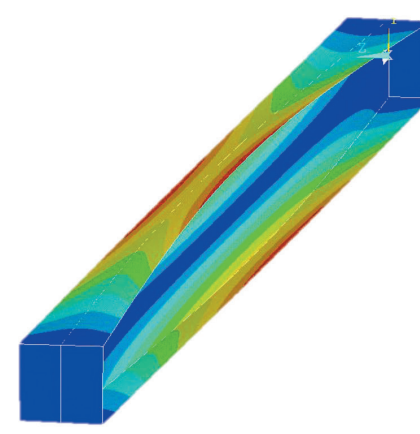

$t_{s t} / t_{w}=0.5$

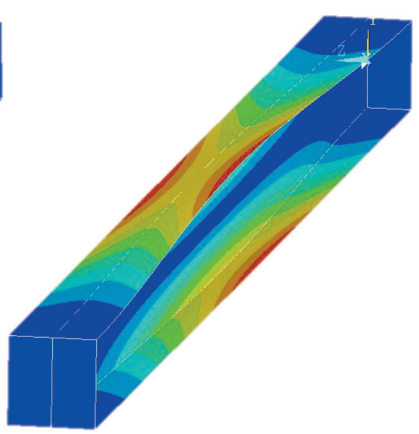

$t_{s t} / t_{w}=10$
Fig. 9 Buckling shapes, $L=6 \mathrm{~m}$, flanges-only connection 


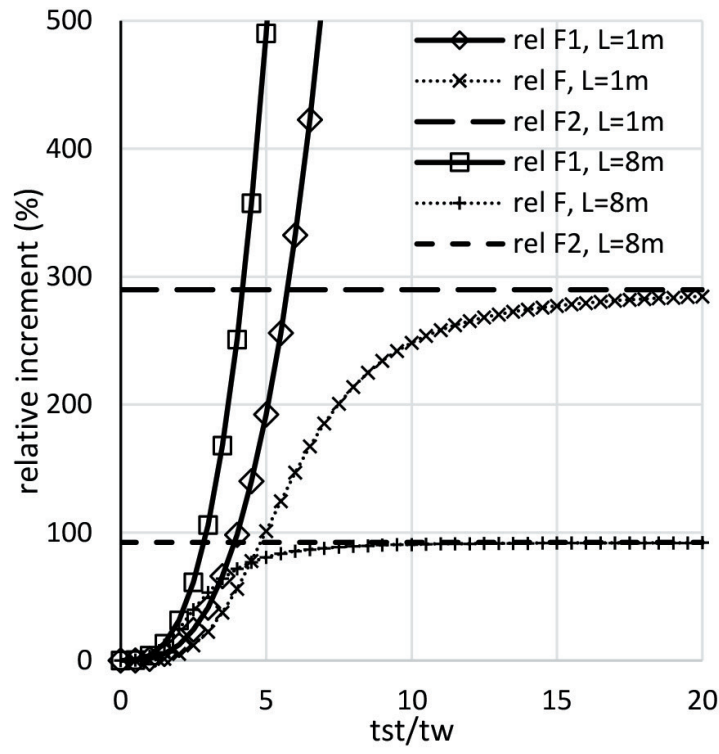

flanges-only connection

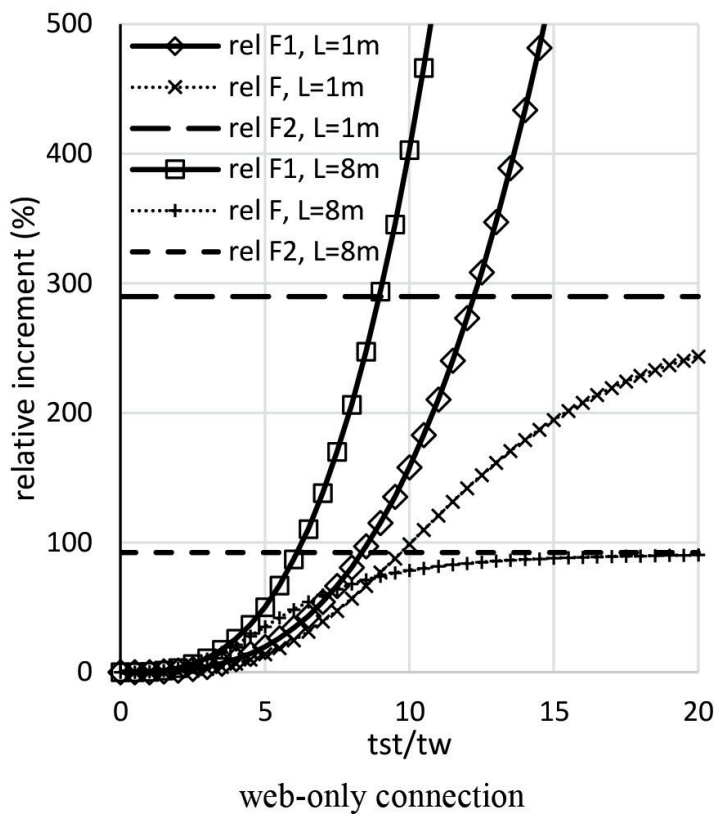

Fig. 10 Relative critical load increments, pinned member with two end-plates

the critical-load-increasing effect of the end-plates with a certain thickness is dependent on the member length. It is also strongly dependent on the stiffener-to-member connection. It is worth noting that the stiffener-to-member connection influences the critical load value through $F_{1}$ only, since the values of $F_{12}$ and $F_{2}$ are independent from the stiffener-to-member connection. (It should also be mentioned that in the case of web-only connection the in-plane shear deformation in the flanges, also known as shear lag effect, has pronounced role in the behavior, but the analysis of this is out of the scope of the current paper.)
In Fig. 4 it was demonstrated that the critical load increment due to a single stiffener is linearly proportional to the inverse of the member length, as predicted by the analytical formula, see Eq. (70). In the case of pinned-pinned column with two end-plates, the analytical formula is complicated, hence it is not obvious how the load increment is related to the member length. As illustrated in Fig. 11, the increment is approx. inversely proportional to the length, but not linearly: in a log-log plot the lines are not perfectly linear, and their tangent is dependent on the end-plate thickness. This tangent increases from 1 to 2 as the end-plate thickness increases from zero to infinity.
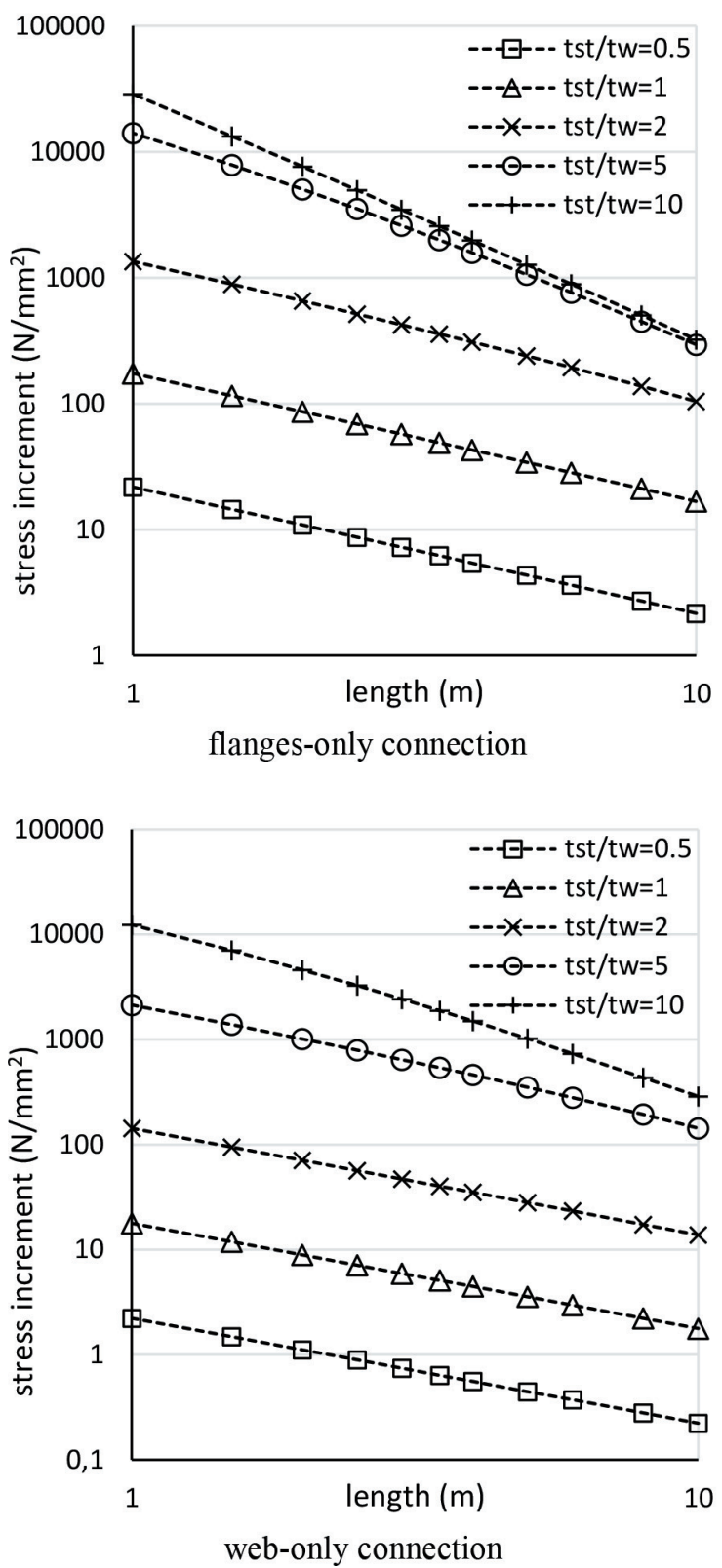

Fig. 11 Relative critical load increments, pinned member with two end-plates 
(For the actual example, in the case of the flanges-only connection the tangent values are approximately 1.00, $1.02,1.05,1.59,1.93$ and 1.99 for $t_{s t} / t_{w}$ values $0.5,1,2,5,10$ and 20, respectively.)

This changing tangent (i.e., changing exponent of $1 / L$ ) is associated with the two major effects of the end-plates (or stiffeners, in general). If the end-plates are very thin, then the longitudinal distribution of the twisting rotations is approximately a half-sine wave, that is the direct effect of the end-plates is dominant, and the direct effect is proportional to $1 / L$. On the other hand, if the end-plates are very thick, then the longitudinal distribution of the twisting rotations is approximately identical to that of a clamped-clamped member, (when the end-plates are hardly deformed, thus their direct effect is negligible,) and in this case the (indirect) effect of the end-plates is materialized in the change of the longitudinal distribution of the twisting rotations; that is why the effect of the end-plates is proportional to $1 / L^{2}$. In between the "very thin" and "very thick" cases the direct and indirect effects are combined, that is why the effect of the end-plates is proportional to $1 / L^{\beta}$, where $\beta$ is between 1 and 2. (It is also worth noting that that the direct effect appears in $F_{1}$, the indirect effect appears in $F_{2}$.)

\section{Conclusions}

In this paper analytical solutions are derived to calculate the critical load of thin-walled column members to pure torsional buckling, by taking the effect of transverse plate elements (termed, for the sake of simplicity, stiffeners) into consideration. The energy method is applied for the derivations. The applied methodology can theoretically be applied to nearly any member and stiffener geometry, however, as proved, for simpler cases the critical force formula can be expressed in closed form.

The analytical solutions demonstrate the two major effects of the stiffeners. A direct effect is due to the deformations of the stiffeners. It can be understood - by adopting the logic of energy method - as follows: the deformed stiffeners increase the strain energy of the structure without changing its external energy, therefore the critical force is increased. The other, indirect effect is that the introduction of stiffeners can (and typically do) modify the longitudinal distribution of the twisting rotations of the member.

The direct effect appears in the critical force formula as an additional term, depending on 4 factors.

- It is linearly proportional to the inverse of the length of the member. Since the Saint-Venant torsional term is independent of the length, while the warping term is proportional to $1 / L^{2}$, it is misleading to represent the effect of the a modified $I_{t}$, or a modified $I_{\omega}$, or an equivalent buckling length.

- It is proportional to the plate stiffness (of the stiffener), that is highly sensitive to the thickness of the stiffener.

- It is dependent on the geometry of the stiffener, as well as on the connection between the stiffener and the main member. In the case of I-section members and simple rectangular stiffeners, it is found that the key factor is whether the stiffener is connected to the flanges, while the web-to-stiffener connection has only small effect.

- The increase of the critical force due to a stiffener is also influenced by the position of the stiffener. More precisely, the effectiveness of the stiffener is dependent on the value of first derivative of the twisting function at the location of the stiffener.

The indirect effect of the stiffeners was demonstrated by the analytical solution of a pinned-pinned column with end-plates. The analytical solution is in accordance with earlier findings and engineering expectations. The endplates work as elastic warping restraints, and the thicker the end-plate, the stronger the warping restraint. The increase of the critical force due to the indirect effect of the end-plates is proportional to $1 / L^{2}$.

When both the direct and indirect effects are present, (as in the case of a pinned-pinned member with end-plates,) the final increase due to the stiffeners is determined by the combination of the two effects. The combination is dependent on the parameters of the problem (e.g., member length, stiffener thickness, etc.). The analytical solution proves that the critical force is bounded: one upper bound is associated with the direct effect, the other upper bound is associated with the indirect effect.

In the paper several numerical examples were presented: the analytical results were compared to results from shell finite element linear buckling analysis. The comparison justifies the analytical solutions, since the tendencies form the two methods are practically identical (at least within the range of validity of the analytical solutions).

Some difference between the finite element and the analytical results were found. In many cases the differences are small, caused by the differences between the underlying assumptions of the beam models (used in the analytical solutions) and the shell models (used in the finite element calculations). In some cases the differences are large. Large differences are experienced when the stiffeners 
significantly modify the longitudinal distribution of the twisting rotations. Relatively large differences may also be caused by the seemingly small differences between the shell-model-based and beam-model-based deformations, i.e., in-plane shear strains, plate-like deformations of the web and flanges of the member, etc., which are allowed in the shell model, but excluded from the beam model. A more formal investigation of these latter differences requires modal buckling analysis. Such modal buckling analysis of thin-walled members with transverse stiffeners will be presented by the authors in a subsequent paper.

\section{References}

[1] Timoshenko, S. P., Gere, J. M. "Theory of Elastic Stability", 2nd ed., McGraw-Hill, New York, NY, USA, 1961.

[2] Vlasov, V. Z. "Thin-walled Elastic Beams", National Science Foundation, Washington, DC, USA, 1961.

[3] Chróścielewski, J., Lubowiecka, I., Szymczak, C., Witkowski, W. "On some aspects of torsional buckling of thin-walled I-beam columns", Computers \& Structures, 84(29-30), pp. 1946-1957, 2006. https://doi.org/10.1016/j.compstruc.2006.08.015

[4] Rao, C. K., Rao, L. B. "Torsional post-buckling of thin-walled open section clamped beam supported on Winkler-Pasternak foundation", Thin-Walled Structures, 116, pp. 320-325, 2017. https://doi.org/10.1016/j.tws.2017.03.017

[5] Szymczak, C., Kujawa, M. "Torsional buckling and post-buckling of columns made of aluminium alloy", Applied Mathematical Modelling, 60, pp. 711-720, 2018. https://doi.org/10.1016/j.apm.2018.03.040

[6] Taras, A., Greiner, R. "Torsional and flexural torsional buckling - A study on laterally restrained I-sections", Journal of Constructional Steel Research, 64(7-8), pp. 725-731, 2008.

https://doi.org/10.1016/j.jcsr.2008.01.019

[7] Gotluru, B. P., Schafer, B. W., Peköz, T. "Torsion in thin-walled cold-formed steel beams", Thin-Walled Structures, 37(2), pp. 127$145,2000$.

https://doi.org/10.1016/S0263-8231(00)00011-2

[8] Bian, G., Peterman, K. D., Torabian, S., Schafer, B. W. "Torsion of cold-formed steel lipped channels dominated by warping response", Thin-Walled Structures, 98(B), pp. 565-577, 2016. https://doi.org/10.1016/j.tws.2015.10.023

[9] Fujii, K., Ohmura, H. "A Study of Rigidity and Strength in Torsion of H-Beam Stiffened with Transverse Stiffeners", Doboku Gakkai Ronbunshu, 1985(356), pp. 289-292, 1985. https://doi.org/10.2208/jscej.1985.356_91
In this paper pure torsional buckling was discussed only, which buckling type has limited practical relevance. However, the applied methodology and also some of the major conclusions can be extended to other types of buckling with torsion, including lateral-torsional buckling, which is the critical behavior type for many beams.

\section{Acknowledgement}

The presented work was conducted with the financial support of the K119440 project of the Hungarian National Research, Development and Innovation Office.

[10] Gunalan, S., Mahendran, M. "Improved design rules for fixed ended cold-formed steel columns subject to flexural-torsional buckling", Thin-Walled Structures, 73, pp. 1-17, 2013. https://doi.org/10.1016/j.tws.2013.06.013

[11] Lindner, J. "Influence of constructional details on the load carrying capacity of beams", Engineering Structures, 18(10), pp. 752-758, 1996. https://doi.org/10.1016/0141-0296(95)00111-5

[12] Santarelli, M., Heinisuo, M., Aalto, A. "Effect of end plates on lateral torsional buckling loads of steel beams in ambient and fire", Rakenteiden Mekaniikka (Journal of Structural Mechanics), 43(2), pp. 73-93, 2010. [online] Available at: http://rmseura.tkk.fi/rmlehti/ 2010/nro2/RakMek_43_2_2010_1.pdf [Accessed: 10 January 2020]

[13] Živner, T. J. "The Influence of Constructional Detail to LateralTorsional Buckling of Beams", Procedia Engineering, 40, pp. 504 $509,2012$. https://doi.org/10.1016/j.proeng.2012.07.134

[14] Takabatake, H. "Lateral buckling of I beams with web stiffeners and batten plates", International Journal of Solids and Structures, 24(10), pp. 1003-1019, 1988. https://doi.org/10.1016/0020-7683(88)90104-7

[15] ANSYS "Release 19.2" [online] Available at: http://storage.ansys. com/doc_assets/release_notes/Release_Notes_192.pdf [Accessed: 10 January 2020]

[16] Ádány, S., Visy, D. "Global buckling of thin-walled columns: Numerical studies", Thin-Walled Structures, 54, pp. 82-93, 2012. https://doi.org/10.1016/j.tws.2012.02.001 